\title{
Diurnal variation and adaptation of the cough response to citric acid in normal subjects
}

\author{
JC POUNSFORD, KB SAUNDERS \\ From the Department of Medicine I, St George's Hospital Medical School, London
}

ABSTRACT The lowest concentrations of citric acid were measured that caused coughing in 10 normal subjects who inhaled successively higher concentrations. Two subjects did not cough at any concentration. In the remaining eight the threshold concentration was significantly higher when measured in the afternoon than it was in the morning $(p<0.05)$. The expected diurnal variation in peak expiratory flow rate (PEFR) was found (significant in the whole group-p $<$ 0.05); but PEFR did not change significantly when measured before and immediately after coughing caused by citric acid inhalations. In a second group of 10 normal subjects two series of citric acid inhalations were given, separated by one hour. The total number of coughs was significantly lower on the second run $(p<0.05)$. Thus diurnal variation and adaptation of the cough response must be taken into account when antitussive drugs are tested.

Cough can be induced in man by inhalation of nebulised citric acid.' Two methods are commonly used; the one most frequently described requires inhalation of varying concentrations of stimulant for a predetermined period, during which the subject attempts to breathe normally. ${ }^{2}$ The concentration at which coughing occurs (the cough threshold) and the number of coughs (the cough response) are recorded. Another method depends on a single breath technique, in which the subject exhales to a known lung volume and then inhales rapidly to total lung capacity (TLC). ${ }^{3}$ Both methods have been reported to give reproducible results. We have modified the second technique, and attempted to answer the following questions: Are there differences in the cough response to citric acid that depend on the time of day when it is measured? If so, in what sense is it related to the diurnal variation in airways resistance in $\operatorname{man}^{4}$ ? Does the cough reflex show adaptation and is the test repeatable after one hour? Can dose-response curves (in terms of number of coughs) be constructed? These questions have not been answered previously and all are relevant to trials of antitussive agents.

Address for reprint requests: Professor KB Saunders, Department of Medicine I, St George's Hospital Medical School, London SW17 ORE.

Accepted 22 February 1985

\section{Methods}

\section{GROUP 1}

Ten healthy volunteers aged $19-40$ years took part in the first group of experiments. None had had an upper respiratory tract infection in the previous eight weeks and none had a history of atopy. They all gave their informed consent to the study, which was approved by the ethical committee of our hospital. Each subject was given four challenges of citric acid, two between 9 am and mid-day and two between 2 and $5 \mathrm{pm}$. Each challenge was done on a separate day, but the exact time of the experiment was not more precisely controlled. The order in which the experiments were conducted was randomised. The citric acid was dissolved in distilled water to a $20 \%$ solution, which was further diluted to the required concentration with normal saline.

After arriving at our laboratory the subjects rested for 15 minutes. They then put on a nose clip and we measured their peak expiratory flow rate (PEFR) with a Wright's peak flow meter. The best of two results was taken as a baseline PEFR. The subjects then breathed normally while sitting in front of a stop clock. After five minutes they were asked to exhale to residual volume (RV). As they approached RV they connected themselves to a $T$ mouthpiece, through one limb of which flowed citric acid nebulised by a Wright's nebuliser at $101 \mathrm{~min}^{-1}$. To the other was attached a rotary spirometer 

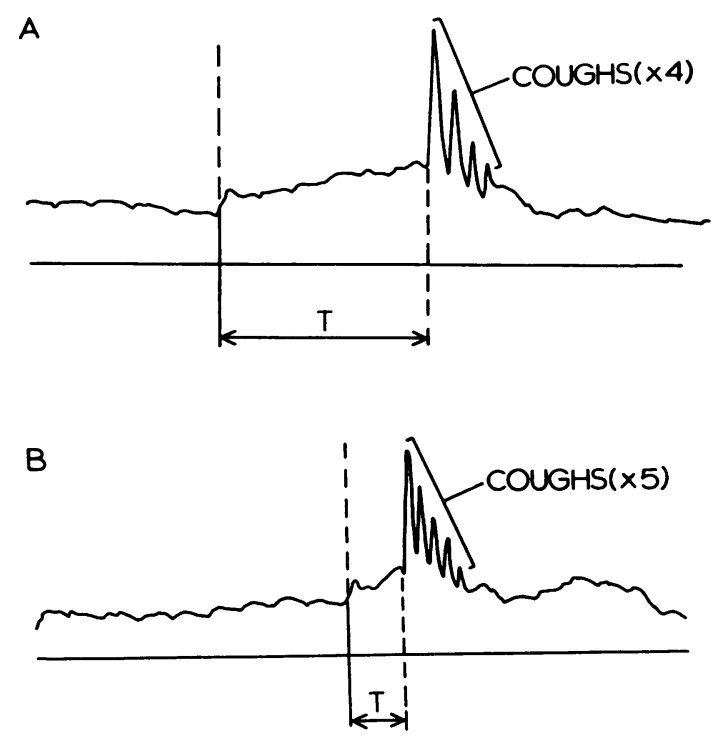

Fig 1 Recording showing calculation of cough index: $(A)$ four coughs with a latency $(T)$ of five seconds and a cough index of 0.8 and $(B)$ five coughs with a latency of 1.3 seconds and a cough index of 3.8 .

(Wright's respirometer) that was open to the air. When they reached residual volume they immediately inhaled to total lung capacity (TLC) in five seconds while observing the stop clock. The volume of inhaled aerosol was therefore about $5 / 60 \times 101$, or $833 \mathrm{ml}$.

After each inhalation we checked the volume of air inhaled, repeated the measurement of PEFR, waited two minutes, and then gave the citric acid at an increased concentration. The citric acid was started at a concentration of $0.5 \%$ and increased in $0.5 \%$ increments to $5 \%$, then in $1 \%$ increments to $10 \%$, and finally in $2.5 \%$ increments to $17.5 \%$. The first concentration at which coughing occurred (the cough threshold) was noted.

\section{GROUP 2}

We selected another 10 healthy volunteers aged 20-38 years, and adapted the technique so that we could measure the time from the start of inhalation to the first cough (the latency of the response) as well as the number of coughs. We divided the number of coughs by the latency and called the result the "cough index" for that inhalation. The cough detector was a mercury strain gauge loosely attached around the subject's neck and connected to a Medelec fibreoptic recorder (fig 1).

The citric acid was started at a concentration of $0.5 \%$ but increased in this protocol by doubling concentrations to a total of $16 \%$, four minutes being
Cough thresholds in group 1 subjects

\begin{tabular}{llc}
\hline Subject No & \multicolumn{2}{l}{ Threshold citric acid concentrations (\%) } \\
\cline { 2 - 3 } & Morning & Afternoon \\
\hline 1 & $2.5,2.5$ & $2.0,2.0$ \\
2 & $3.5,1.5$ & $5.0,5.0$ \\
3 & $3.5,3.0$ & $3.0,7.0$ \\
4 & $2.5,2.0$ & $7.0,3.0$ \\
5 & $3.0,3.0$ & $3.0,3.5$ \\
6 & $3.0,3.0$ & $5.0,3.0$ \\
7 & $2.5,3.0$ & $5.0,3.0$ \\
8 & $3.5,1.5$ & $10.0,4.5$ \\
\hline
\end{tabular}

allowed between each inhalation. The cough threshold was again noted. The experiment was repeated one hour later to determine whether the cough response had altered. The whole study (that is, two series of citric acid inhalations separated by a one hour interval) was repeated on another day at the same time of day to see whether the results were reproducible.

\section{Results}

\section{GROUP 1}

Two of the 10 subjects did not cough at any citric acid concentration on any of the four days. Threshold values for the remaining eight are shown in the table. There was agreement within $2 \%$ for the two morning results, but variability was greater in the afternoon.

We took for further analysis the means of the two morning and two afternoon thresholds, and the means of the two morning and the two afternoon baseline PEFRs. Of these eight subjects, seven had a higher threshold and six a higher PEFR in the afternoon (fig 2). The overall mean morning threshold ( $2.84 \%$ citric acid (1 SD $0.33 \%)$ ) was significantly less than the overall mean afternoon threshold $(4.44 \%(1.54 \%) ; \mathrm{p}<0.05$, paired $t$ test). The overall mean morning baseline PEFR ( $507(87) 1 \mathrm{~min}^{-1}$ ) was significantly less than the overall mean afternoon baseline PEFR (530 (89) $1 \mathrm{~min}^{-1} ; \mathrm{p}<0.05$, paired $t$ test).

The mean value of all cough thresholds obtained on day 1 was $4.13 \%(1.46 \%)$; on day $2,3.43 \%$ $(2.73 \%)$; on day $3,3.44 \%(1.74 \%)$; and on day 4 , $3.18 \%(1.06 \%)$. Although there was a trend for the mean thresholds to decrease as the experiments proceeded this decrease was not significant at the 5\% level (analysis of variance).

We noted baseline PEFR (before any citric acid was given), subthreshold PEFR (just before the inhalation of citric acid that caused coughing), and postthreshold PEFR (after the first bout of induced coughing). No significant differences were found (mean values $518(87), 514$ (76), and $513(80)$ $1 \mathrm{~min}^{-1}$ ). 


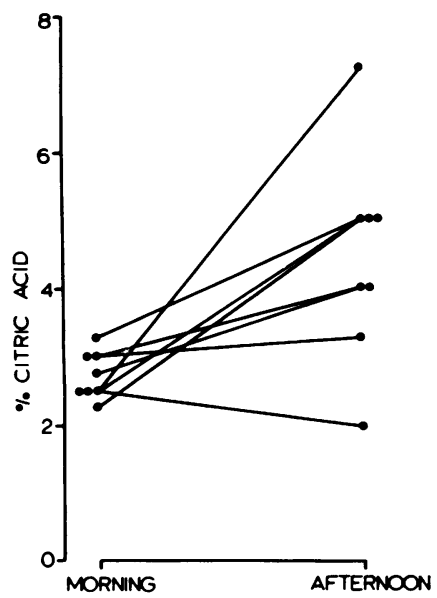

GROUP 2

We took for further analysis cumulated sums of the coughs obtained in each citric acid run from 0 to $16 \%$ for each subject, two runs on each of two days, and the similarly cumulated sums of the "cough indices."

On day 1 the number of coughs and the total of "cough indices" were significantly less on the second run, one hour after the first $(\mathrm{p}<0.01$ and $<0.05$ respectively, Wilcoxon's signed rank test). On day 2 the same result was obtained ( $p<0.01$ for both criteria). Individual results for day 1 are shown in figure 3.

Among 20 comparisons the threshold increased in nine and was unchanged in 11 after the one hour interval. This trend to increase was not significant, either by paired $t$ test or by Wilcoxon's signed rank test.

\section{Discussion}

The cough reflex is stimulated by sensitisation of rapidly adapting receptors located in the larynx and bronchial tree. ${ }^{56}$ These receptors ramify between individual epithelial cells and afferent nerve signals travel in myelinated nerve fibres in the vagus nerve to the cough centre of the brainstem. The receptors are of two types: those sensitised by touch, which
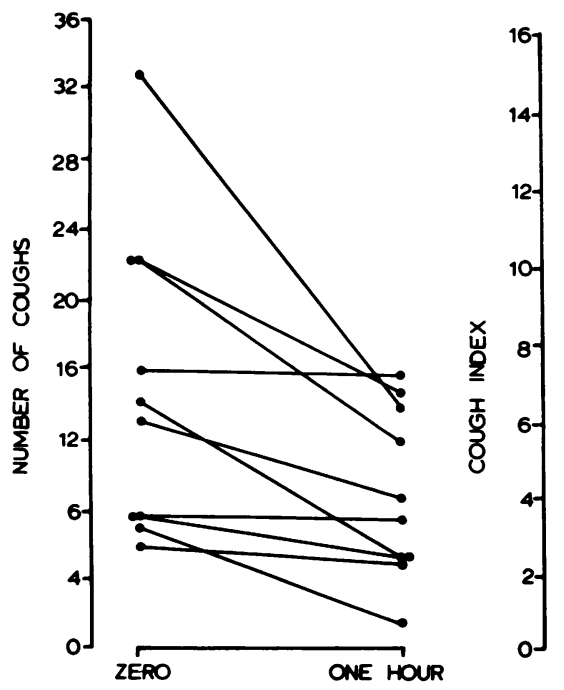

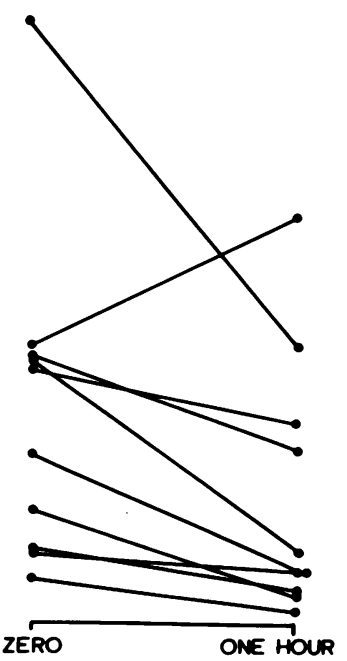

Fig 3 Cumulated coughs and cough indices on day 1 in 10 subjects showing adaptation of the response after one hour $(p<0.01$ (coughs) and $p<0.05$ (cough index), Wilcoxon signed rank test) without drug intervention. 
are found in the trachea and at the divisions of major airways ${ }^{5}$; and "irritant receptors," which are sensitised by inhaled irritants and bronchoconstriction $^{7}$ and are located in smaller airways down to terminal bronchioles. Citric acid probably stimulates these irritant receptors, but this cannot be shown conclusively in man. Rapidly adapting receptors are also found in the larynx, ${ }^{8}$ and it might be stimulation of these by citric acid that induces cough in man.

To maintain a reproducible stimulus to the airways we administered citric acid aerosol using a single breath technique. The alternative method, in which a subject attempts to breathe normally while inhaling citric acid, has the disadvantage that any irritation or coughing induced would stimulate an altered pattern of inhalation and deposition of the aerosol would be changed. Furthermore, the deep inhalation which occurs before coughing would cause stimulation and recruitment of irritant receptors. These might modify the response to such an extent that no other inhalation of citric acid would be directly comparable unless the pattern of coughing and the alteration in lung volumes induced were identical. Unlike Empey, ${ }^{3}$ we asked our subjects to inhale from RV to TLC over five seconds rather than inhale as rapidly as possible. This was because of the effect of rapid inhalation on smooth muscle receptors and because rapid inhalation has been reported to cause bronchodilatation, ${ }^{9}$ which is reduced by a slower inhalation.

In the first study we showed that cough threshold was systematically and significantly higher in the afternoon. Consequently it is important that studies of antitussive drugs in an individual should be done at the same time of day. We did not in this study have a reliable method of counting coughs, and based our conclusion on cough threshold alone. In the second study we recorded and counted the coughs, hoping to construct a dose-response curve, and for the same reason we used logarithmic dosage increments. Plausible dose-response curves were obtained in only three of 10 subjects. We therefore abandoned the dose-response approach, and took the total number of coughs. This was sufficient to show that the cough response to citric acid is diminished when repeated an hour later, even without any pharmacological intervention.

The switch to logarithmic dose increments increases the interval between successive doses around the normal threshold, and makes determination of the threshold necessarily less precise. We suspect that this accounts for the finding that, despite a diminution in cough response, the threshold showed only a non-significant trend to increase.

We had gone to some pains, as described above, to control the inhaled volume of nebulised citric acid. Some subjects nevertheless coughed during the inhalation, before reaching TLC, and therefore got a smaller dose. We tried to compensate for this by weighting positively the number of coughs recorded during short inhalations. This was done by dividing the number of coughs by the latency of the response. The resulting "cough index" gave the same result as we obtained by using the total number of coughs. We wished to know whether the citric acid inhalations themselves changed airways resistance. The PEFR measurements we used were sufficiently sensitive to detect the expected normal diurnal variation $^{4}$ in the calibre of airways, but showed no effect of citric acid in concentrations just sufficient to cause coughing and no effect of the coughing itself. Airways resistance measurements by plethysmography might have shown a significant change, but we conclude that any effect of citric acid on airways resistance must be, at the most, small.

We showed adaptation of the response over the course of one hour. It is entirely possible that adaptation occurs over a shorter period, even during the course of a single series of citric acid inhalations. This would not affect our conclusions since we used a standard procedure in every case. Over a longer time scale threshold tended to decrease, though not significantly, over the four days of the group 1 experiments. Again, this would not disturb our demonstration of diurnal variation, since morning and afternoon experiments were performed in randomised order over that time period.

The adaptation of the response to citric acid shown in our second set of experiments could occur at receptor or brainstem level. None of our subjects was able to suppress a desire to cough but they frequently reported a minor sense of irritation in the throat at concentrations below the cough threshold. The irritant receptors are rapidly adapting ones and increased concentrations of acid produced more coughs, which implied that the receptors had returned to their resting potentials before additional stimulant was given. The change that occurred after one hour is unlikely to be an entirely local effect and some central adaptation is probable. A tenable explanation for adaptation at local level is an increase in mucus production stimulated by the presence of an irritant on the bronchial mucosa, as shown by Phipps and Richardson. ${ }^{10}$

We have shown an appreciable difference between the cough thresholds obtained in the morning and in the afternoon. The lower threshold in the morning is unlikely to be due to the mechanical effect of any excess bronchial mucus in the morning, particularly in normal subjects, none of whom reported throat clearing or morning coughing as a 
regular habit. Food and other ingested materials could alter the sensitivity of pharyngeal cough receptors, although these have not been reliably confirmed to exist in man and our subjects were not instructed to fast before the study. The highest cough thresholds were obtained late in the afternoon and were not related to a meal. Normal nonsmokers frequently cough when they walk into a smoke filled room but tolerance to the irritating atmosphere develops rapidly. It is perhaps not surprising that the cough response should alter during the day given the variety of atmospheric pollutants to which we are exposed.

We conclude, firstly, that tests of cough response provoked by nebulised inhaled citric acid should be performed at the same time of day in individual subjects and, secondly, that in assessments of antitussive agents conclusions should not be based on comparisons of citric acid provocation before and after drug or placebo but on provocations after drug and placebo given on different days.

We would like to thank the South West Thames Regional Health Authority, which funded JCP with a locally organised research grant, and BoehringerIngelheim Ltd for additional financial support.

\section{References}

1 Bickerman HA, Barach AL. The experimental produc- tion of cough in human subjects induced by citric acid aerosols. Am J Med Sci 1954;228:156-63.

2 Bickerman HA, German E, Cohen BM, Itkin SE. The cough response of healthy human subjects stimulated by citric acid aerosol. Am J Med Sci 1957;234:191205.

3 Empey DW, Laitinen LA, Young GA, Bye CE, Hughes DTD. Comparison of the antitussive effects of codeine phosphate $20 \mathrm{mg}$ dextromethorphan $30 \mathrm{mg}$ and noscapine $30 \mathrm{mg}$ using citric acid-induced cough in normal subjects. Eur J Clin Pharmacol 1979;16:3937.

4 Hetzel MR, Clark TJH. Comparison of normal and asthmatic circadian rhythm in peak expiratory flow rate. Thorax 1980;35:732-8.

5 Widdicombe JG. Respiratory reflexes from the trachea and bronchi of the cat. J Physiol 1954;123:55-70.

6 Widdicombe JG. Receptors in the trachea and bronchi of the cat. J Physiol 1954;123:71-104.

7 Mills JE, Sellick H, Widdicombe JG. Activity of lung irritant receptors in pulmonary microembolism, anaphylaxis and drug-induced bronchoconstrictions. $J$ Physiol 1969; 203:337-57.

8 Boushey HA, Richardson PS, Widdicombe JG, Wise JCM. The response of laryngeal afferent fibres to mechanical and chemical stimuli. J Physiol 1974;240: 153-75.

9 Beaupré A, Orehek J. Factors influencing the bronchodilator effect of a deep inspiration in asthmatic patients with provoked bronchoconstriction. Thorax 1982;37:124-8.

10 Phipps RJ, Richardson PS. The effects of irritation at various levels of the airway upon tracheal mucus secretion in the cat. J Physiol 1976;261:563-81. 